RALF WASSMUTH und HEINO ALPS

\title{
Erfassung der Futteraufnahme im Rahmen der Stationsprüfung potentieller Besamungsbullen
}

\begin{abstract}
Summary
Title of the paper: Recording of feed intake in stationary testing of potential AI bulls

This study was performed in order to estimate correlations between feed intake, eating time and daily gain in young bulls and in order to estimate genetic parameters for feed intake in successive testing periods aiming at reduction of costs for testing. Data were collected from 269 station tested potential Al bulls of German Holstein. In the testing period beginning with the $112^{\text {th }}$ and ending with the $312^{\text {th }}$ day of life, bulls consumed $5.1 \mathrm{~kg}$ roughage in 125 minutes per day and increased their weight by $1,300 \mathrm{~g}$ daily. The heritability of feed intake was 0.42 , of eating behaviour 0.40 and of daily gain 0.62 . Between feed intake and eating behaviour no relationship could be observed. Hence, an indirect measurement of feed intake is not possible. The high genetic correlation between feed intake and daily gain of 0.96 is no reason to replace feed intake recording because of a promising relationship between feed intake and health of dairy cows. Because of a delayed start of $32 \%$ of all tested bulls a shortening of the test period should be orientated to the last testing periods. In the last testing period, the heritability of feed intake was 0.65 and the genetic correlation between feed intake in the last and in the whole test period was high with 0.88 . Hence, the test period could be shortened favouring intake measurements between $263^{\text {rd }}$ and $312^{\text {th }}$ day of life.
\end{abstract}

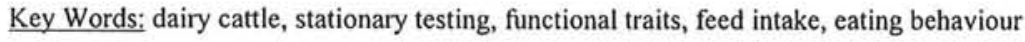

\section{Zusammenfassung}

Das Ziel der Untersuchung war, Möglichkeiten der Kostensenkung durch eine zeitliche Verkürzung der stationären Futteraufnahmeprüfung und durch eine Erfassung des Futteraufnahmeverhaltens zu prüfen. Es konnten Daten aus der stationären Prüfung von 269 potentiellen Besamungsbullen (Deutsche Holstein) einbezogen werden. Im Prufabschnitt 112. bis 312. Lebenstag nahmen die Jungbullen durchschnittlich $5,1 \mathrm{~kg}$ Grundfutter in 125 Minuten pro Tag auf und erhø̆hten ihr Körpergewicht um $1.300 \mathrm{~g}$ tăglich. Die Heritabilităt der Futteraufnahme betrug 0,42, die der Futteraufnahmedauer 0,40 und die der täglichen Zunahme 0,62. Zwischen der Futteraufnahme und der Futteraufnahmedauer bestand keine Beziehung, so daß eine indirekte Erfassung der Futteraufnahme nicht möglich ist. Die hohe genetische Korrelation zwischen der Futteraufnahme und der tăglichen Zunahme von 0,96 sollte jedoch nicht zum Verzicht auf die Futteraufnahmeprufung fuhren, da letztere eine vielversprechende Beziehung zur Gesundheit von Milchkuhen besitzt. Aufgrund der verspäteten Anlieferung von $32 \%$ aller Kălber sind nur die späteren Teilabschnitte für eine Verküzung der Futteraufnahmeprüung geeignet. Im letzten 50-Tageabschnitt des Gesamtabschnittes erreichte die Futteraufnahme eine Heritabilităt von 0,65 und eine genetische Korrelation zur Futteraufnahme im Gesamtabschnitt von 0,88 . Somit ist ein größerer Informationsverlust nicht zu erwarten, wenn die Futteraufnahmeprüfung entsprechend verkürzt wird.

Schlüsselwörter: Milchrinder, Stationsprüfung, funktionale Merkmale, Futteraufnahme, Futteraufnahmeverhalten

1. Einleitung

Aus züchterischer Sicht basiert eine effiziente Produktion auf der Verbesserung von Produktionseigenschaften und der Berücksichtigung funktionaler Merkmale bei Selektionsentscheidungen (GROEN et al., 1997). In der Milchrinderzucht kommt als 
Produktionseigenschaft der Steigerung der Milchleistung die größte Bedeutung zu während die Fleischleistung aus dem Zuchtziel gestrichen wurde. Vor dem Hintergrund der in der Literatur nachgewiesenen genetischen Antagonismen zwischen der Milchleistung einerseits und der Gesundheit sowie der Fruchtbarkeit andererseits (MÄNTYSAARI et al., 1991; SIMIANER et al., 1991; GROEN et al., 1994; URIBE et al., 1995; PRYCE et al., 1997) ist es dringend erforderlich, über die Einbeziehung funktionaler Merkmale in das Zuchtziel, steigenden Erkrankungsfrequenzen bei züchterischer Verbesserung der Milchleistung entgegenzuwirken.

Die züchterische Verbesserung funktionaler Merkmale hat die Steigerung der Gesundheit und damit die Reduktion der Produktionskosten zum Ziel. Darüber hinaus kann die Verbraucherakzeptanz der erzeugten Produkte verbessert werden, wodurch Marktanteile gefestigt oder neu hinzu gewonnen werden können.

In den skandinavischen Ländern werden Merkmale, die die Gesundheit eines Tieres beschreiben, direkt in das Zuchtziel einbezogen (ANONYMUS, 1996). Die Basis hierfür ist ein zentral geführtes Melderegister, das auf einer landesweiten Erfassung der Erkrankungen von Milchkühen basiert (ANDERSEN, 1991). In Populationen, in denen solche Erfassungssysteme nicht existieren, werden indirekte Merkmale der Gesundheit im Zuchtziel berücksichtigt (ANONYMUS, 1996). Es gilt, weitere Indikatoren der Gesundheit als Hilfsmerkmale für die züchterische Verbesserung dieses Komplexes zu entwickeln.

Nach WASSMUTH (1999) liefert die Futteraufnahmeprüfung potentieller Besamungsbullen gute Hinweise auf die Gesundheit ihrer milchleistenden Töchter. Die vielversprechendste genetische Beziehung wurde zwischen der Futteraufnahme der Bullen und der Ketosefrequenz ihrer Töchter geschätzt. Hieraus kann gefolgert werden, dass die Selektion von Besamungsbullen mit hoher Futteraufnahme zu einer geringeren Ketosefrequenz in der Milchkuhpopulation führt. Die Selektion auf hohe Futteraufnahme ist mit der züchterischen Steigerung der Milchleistung zu vereinbaren, da von einer leicht positiven genetischen Korrelation zwischen beiden Merkmalen auszugehen ist (BRANDT et al., 1985; VAN ARENDONK et al., 1991; JENSEN et al., 1995; WASSMUTH, 1999). Allerdings fuhrt die leicht positive Korrelation zur Futteraufnahme bei Selektion auf höhere Milchleistung nicht zu ausreichend hohen korrelierten Selektionserfolgen in der Futteraufnahme (WASSMUTH, 1999).

Darüber hinaus ist die Selektionswürdigkeit der Futteraufnahme durch eine ausreichend hohe Heritabilität (NIEUWHOF et al., 1992; JENSEN et al., 1995; JAKOBSEN, 1995; MADSEN et al., 1995; FAN et al., 1995; WASSMUTH, 1999; POTTHAST, 1999) und genetische Varianz (WASSMUTH, 1999; POTTHAST, 1999) gegeben.

Die Erfassung der Futteraufnahme ist auf die stationäre Prüfung beschränkt und wird hauptsächlich bei der Prüfung potentieller Besamungsbullen durchgeführt (WASSMUTH und ALPS, 1995). Bei der stationären Eigenleistungsprüfung in Deutschland können 3 verschiedene Futteraufnahmeprüfungen voneinander unterschieden werden (Tab. 1).

Das erste Verfahren wird in Eickelborn und Neu-Ulrichstein angewendet. Es werden Wiegetröge zur täglichen Messung der Grundfutteraufnahme verwendet und als Grundfutter praxisnah eine Totale Mischration bzw. Maissilage eingesetzt. 
Dem Nachteil, der begrenzten Standardisierbarkeit bezüglich des Nährstoff- und Energiegehalts der Maissilage, wird in Echem mit dem Einsatz aufgeschlossener Strohcobs (Natronlauge) begegnet. Die tierindividuelle Strohcobaufnahme wird über eine modifizierte Abruffütterungsanlage, wie sie in der Milchviehhaltung zur Verabreichung von Kraftfutter eingesetzt wird, gemessen. Das zweite Verfahren zur Messung der Futteraufnahme basiert zwar auf einem vom Praxisfutter abweichenden Grundfutter (Strohcobs), es muß allerdings nicht zwangsläufig zu einer anderen Rangierung der geprüften Bullen im Vergleich zur Prüfung in Eickelborn und Neu-Ulrichstein führen. Diesen Schluß lassen die Ergebnisse von POTUCEK (1990) zu. Demnach ist das Auftreten von Genotyp $\times$ Umwelt-Interaktionen verursacht durch Unterschiede in der Grundfutterzusammensetzung nicht zu erwarten.

Die dritte Art der Futteraufnahmeprüfung wird in Ruhwinkel durchgeführt. Hier wird sie nicht direkt, sondern indirekt über die Erfassung des Futteraufnahmeverhaltens und der täglichen Zunahme geschätzt (STAMER et al., 1997). Die Meßtechnik ist dabei kostengünstiger als bei direkter Messung.

Tabelle 1

Prüfabschnitt, Messung der Futteraufnahme und Prüfdiăt in deutschen Prüfstationen (modifiziert nach WASSMUTH und ALPS, 1995) (Test period, recording of feed intake and test diet in German testing stations)

\begin{tabular}{lccc}
\hline Station & $\begin{array}{c}\text { Prüfabschnitt } \\
\text { (Lebenstage bzw. Lebend- } \\
\text { gewicht) }\end{array}$ & $\begin{array}{c}\text { Messung der Futterauf- } \\
\text { nahme }\end{array}$ & $\begin{array}{c}\text { Prïfdiät } \\
\text { (Grundfutter) }\end{array}$ \\
\hline $\begin{array}{l}\text { Eickelborn } \\
\text { Neu-Ulrichstein }\end{array}$ & $\begin{array}{c}125 \mathrm{~kg}-450 \mathrm{~kg} \\
112 .-312 .\end{array}$ & $\begin{array}{c}\text { Wiegetröge } \\
\text { Wiegetröge }\end{array}$ & $\begin{array}{c}\text { Totale Mischration } \\
\text { Maissilage }\end{array}$ \\
\hline Echem & $130 .-330$. & Modifizierter Kraftfutter- & Strohcobs \\
Ruhwinkel & $100 .-365$. & Futteraufnahmeverhalten & Mais- und Grassilage \\
\hline
\end{tabular}

Im vorliegenden Beitrag wurde untersucht, ob die Futteraufnahmeprüfung durch eine Verkürzung der Prüfdauer oder eine indirekte Erfassung kostengünstiger gestaltet werden kann. Hierzu wurden genetische Korrelationen zwischen der Futteraufnahme in aufeinanderfolgenden Prüfabschnitten und zwischen der Futteraufnahme einerseits sowie Merkmalen des Futteraufnahmeverhaltens und der täglichen Zunahme andererseits geschätzt.

2.

Material und Methoden

2.1. Datenmaterial

Die Daten stammen von 269 potentiellen Besamungsbullen, die von 1993 bis 1999 in der Station Neu-Ulrichstein geprüft wurden. Neben 215 schwarzbunten DeutschenHolstein-Bullen von 81 Vätern konnten 54 rotbunte Deutsche Holstein Bullen von 31 Vätern in die Untersuchung einbezogen werden. Die Prüfung erfolgte vom 112. bis 312. Lebenstag und die Bullen erhielten Maissilage mit einem Trockensubstanzgehalt von ca. $32 \%$ ad libitum. Zusätzlich wurden rationierte Kraftfuttergaben nach Alter verabreicht. Eine Haltungsgruppe bestand aus 4 Bullen, die freien Zugang zu 2 Wiegetrögen besaßen.

Die aufgenommene Menge an Maissilage wurde automatisch mittels der Wiegetröge bei jedem Futterbesuch erfasst und in Kilogramm (kg) Trockensubstanz pro Tag tier- 
individuell berechnet.

Zur Beschreibung des Futteraufnahmeverhaltens wurde die Dauer der Futteraufnahme an den Wiegetrögen automatisch aufgenommen und in Minuten pro Tier und Tag angegeben. Die Aufenthaltsdauer an den Trögen ohne Futteraufnahme wurde nicht mit einbezogen.

Die gesamte Prüfperiode von 200 Tagen wurde in 4 aufeinanderfolgende 50-Tagesabschnitte aufgeteilt. Die Futteraufnahme sowie die Futteraufnahmedauer wurden anhand der Tagesdatensätze für den Gesamtabschnitt und die 4 Teilabschnitte berechnet.

Da das Anlieferungsalter von 38 bis 160 Tage schwankte, wurden die Merkmale des Gesamtabschnittes auf einen einheitlichen Prüfbeginn bei 112 Tagen mittels der linearen Regression korrigiert. In den aufeinanderfolgenden 50-Tagesabschnitten wurde die Leistung von Bullen nur dann berechnet, wenn Beobachtungen von mindestens 40 Einzeltagen vorlagen.

Basierend auf den monatlichen Wiegungen der Bullen wurde die tägliche Zunahme mittels Interpolation für den Gesamtabschnitt und die 4 Teilabschnitte in Gramm (g) pro Tag berechnet.

Alle Merkmale waren annähernd normalverteilt.

\subsection{Statistische Methoden}

Die statistische Aufbereitung der Daten wurde mit dem Programmpaket SAS (SAS INSTITUTE INC., 1990) durchgeführt. Über die darin enthaltenen Prozeduren GLM und MIXED wurden fixe und zufällige Effekte auf Signifikanz geprüft.

Varianz- und Kovarianzkomponenten wurden mit dem REML-Algorithmus (NEUMAIER und GROENEVELD, 1998) des Programmpaketes VCE 4.0 von GROENEVELD und GARCIA CORTÉS (1998) geschätzt. Die Verwandtschaftsmatrix wurde aufgestellt, indem bis zu 6 Generationen zurückverfolgt wurden. Tiere mit nur einer Verknüpfung (,single linked animals") wurden nicht einbezogen.

Die Schätzung der Heritabilitäten erfolgte univariat. Bivariate Analysen dienten der Schätzung genetischer Korrelationen.

Das folgende lineare Modell kam für die statistischen Auswertung der Daten zur Anwendung:

$y_{i j k l}=\mu+L_{i}+J S_{j}+b^{*} a_{i j k l}+t_{k}+e_{i j k l}$

wobei gilt: $y_{i j k l}=1$-te Beobachtung, $\mu=$ Gesamtmittel, $L_{i}=$ fixer Effekt der Linie $(i=1$ für schwarzbunte Deutsche Holstein, $\mathrm{i}=2$ für rotbunte Deutsche Holstein), $\mathrm{JS}_{\mathrm{j}}=$ fixer Effekt des Geburtsjahres $\times$ Geburtssaison ( $j=1$ für das 2. Quartal 1993 bis $j=20$ für das 1. Quartal 1998), wobei die Geburtssaison in Anlehnung an das erste, zweite, dritte und vierte Quartal eines jeden Jahres vergeben wurde, $b=$ lineare Regression auf das Anlieferungsalter, $a_{i j k l}=$ Anlieferungsalter des Bullen in Tagen, $t_{k}=$ zufälliger genetischer Effekt des Bullen $\mathrm{k}$ und $\mathrm{e}_{\mathrm{ijkl}}=$ zufälliger Restfehler.

$\mathrm{Da}$ das Anlieferungsalter den Einfluß der Herde, in der die Bullen bis zum Beginn der Prüfung aufgezogen wurden, repräsentiert, wurde es als Kovariable in das statistische Modell aufgenommen. 
3. Ergebnisse

3.1 Genetische Parameter für Merkmale im Gesamtabschnitt

Zwischen dem 112. und 312. Lebenstag nahmen die Jungbullen täglich 5,1 kg Trockenmasse über Maissilage auf und der Variationskoeffizient betrug 10,6\%. Sie benötigten dafür durchschnittlich 125 Minuten pro Tag und erreichten eine tägliche $\mathrm{Zu}$ nahme von $1.300 \mathrm{~g}$ (Tab. 2). Die rotbunten Deutschen Holstein-Bullen nahmen geringfügig weniger Futter in etwas kürzerer Zeit auf als die Schwarzbunten, erzielten aber ähnlich hohe tägliche Zunahmen. Die Extremwerte deuten eine große Variation der Merkmalsausprägung an.

Die Heritabilitäten für die Futteraufnahme, die Futteraufnahmedauer und die tägliche Zunahme betragen $0,42,0,40$ bzw. 0,63 (Tab. 2). Aufgrund der niedrigen Standardfehler muß gefolgert werden, dass die Heritabilitätsschätzwerte signifikant sind.

Tabelle 2

Mittelwert (Standardabweichung ${ }^{1}$ als Subskript), Minimum und Maximum sowie Heritabilităt (Standardfehler als Subskript) fur die Futteraufnahme, die Futteraufnahmedauer und die tägliche Zunahme potentieller Besamungsbullen der Rasse Deutsche Holstein (Mean (standard deviation ${ }^{1}$ in subscript), minimum, maximum and heritability (standard error in subscript) of feed intake, eating time and daily gain in potential Al bulls of the breed German Holstein)

\begin{tabular}{|c|c|c|c|c|c|c|c|c|c|}
\hline \multirow[t]{2}{*}{ Merkmal } & \multirow{2}{*}{\multicolumn{2}{|c|}{ Gesamtmittel }} & \multicolumn{4}{|c|}{ Deutsche Holstein } & \multirow{2}{*}{$\begin{array}{l}\text { Minimum - } \\
\text { Maximum }\end{array}$} & \multicolumn{2}{|c|}{ Heritabilităt } \\
\hline & & & Schwa: & bunt & Rotbun & & & & \\
\hline Anzahl Bullen & 269 & & 215 & & 54 & & & . & \\
\hline $\begin{array}{l}\text { Futteraufnahme } \\
\left(\mathrm{kg} \mathrm{TM} / \mathrm{T}^{2} \mathrm{gg}\right)\end{array}$ & 5,1 & 0.54 & 5,12 & 0.63 & 4,87 & 0.67 & $3,2-6,7$ & 0,42 & 0.20 \\
\hline $\begin{array}{l}\text { Futteraufnahmedauer } \\
\text { (Minuten/Tag) }\end{array}$ & 125 & 17 & 126 & 20 & 124 & 17 & $78-191$ & 0,40 & 0,15 \\
\hline Tägliche Zunahme (g/Tag) & 1.300 & 125 & 1.300 & 162 & 1.297 & 137 & $560-1.681$ & 0,63 & 0,20 \\
\hline
\end{tabular}

Innerhalb des Gesamtabschnittes ergaben sich die in Tabelle 3 aufgefuhrten Merkmalsbeziehungen. Eine hohe genetische Korrelation von 0,96 wurde zwischen der Futteraufnahme und der täglichen Zunahme geschätzt. Aufgrund des geringen Standardfehlers ist der Schätzwert als signifikant anzusehen. Dagegen wurde für die genetische Korrelation zwischen der Futteraufnahme und der Futteraufnahmedauer ein statistisch nicht signifikanter Schätzwert von $-0,12$ berechnet.

\section{Tabelle 3}

Genetische (oberhalb der Diagonalen) und phănotypische (unterhalb der Diagonalen) Korrelationen (Standardfehler als Subskript) zwischen der Futteraufnahme, der Futteraufnahmedauer und der tảglichen Zunahme potentieller Besamungsbullen (Genetic (above diagonal) and phenotypic (below diagonal) correlations (standard error in subscript) between feed intake, eating time and daily gain in potential AI bulls)

\begin{tabular}{|c|c|c|c|c|c|c|}
\hline \multicolumn{2}{|c|}{ Merkmal } & \multirow[t]{2}{*}{$1^{1}$} & \multicolumn{2}{|l|}{2} & \multicolumn{2}{|l|}{3} \\
\hline 1 & Futteraufnahme (kg TM²/Tag) & & $-0,12$ & 0,33 & 0,96 & 0,10 \\
\hline 2 & Futteraufnahmedauer (Minuten/Tag) & 0,12 & & & $-0,23$ & 0,25 \\
\hline 3 & Tăgliche Zunahme (g/Tag) & 0,70 & 0,20 & & & 0,20 \\
\hline
\end{tabular}

T'Die Ziffern stehen fur die Merkmale in der 1. Spalte. ${ }^{2}$ Trockenmasse

\subsection{Genetische Parameter für die Futteraufnahme in Teilabschnitten}

Im 1. Teilabschnitt, der mit dem 112. Lebenstag begann und mit dem 162. endete, konnte nicht für alle Jungbullen die Futteraufnahme berechnet werden, da nur 182 von 
269 Tieren (68\%) rechtzeitig zur Prüfung angeliefert wurden (Tab. 4). Auch zu Beginn des 2. Teilabschnittes waren noch nicht alle Tiere in der Station, wie Tabelle 4 zeigt.

Vom 1. bis zum 4. Teilabschnitt stieg die Futteraufnahme nahezu linear von $3,4 \mathrm{~kg}$ auf $6,5 \mathrm{~kg}$ TM und die phänotypische Standardabweichung von 0,47 auf 0,75 . Der Variationskoeffizient zeigte eine leicht sinkende Tendenz von $13,8 \% \mathrm{im} 1$. Teilabschnitt auf 11,5\% im letzten Abschnitt.

Die Heritabilitäten variierten sehr stark zwischen den verschiedenen Abschnitten. Für den 4. Teilabschnitt wurde eine Heritabilität der Futteraufnahme von 0,65 geschätzt. Die genetische Standardabweichung betrug 0,60 (Tab. 4).

\section{Tabelle 4}

Anzahl gepriffter Bullen (n), Mittelwert, phănotypische Standardabweichung ( $\left.\sigma_{\mathrm{P}}\right)$, Variationskoeffizient (VK), Heritabilităt $\left(h^{2}\right)$ und genetische Standardabweichung $\left(\sigma_{A}\right)$ fur die Futteraufnahme in aufeinanderfolgenden Prüfabschnitten potentieller Besamungsbullen (Number of tested bulls (n), mean, phenotypic standard deviation ( $\sigma_{p}$ ), coefficient of variance (VK), heritability $\left(\mathrm{h}^{2}\right)$ and genetic standard deviation $\left(\sigma_{\mathrm{A}}\right)$ of feed intake in successive test periods in potential AI bulls)

\begin{tabular}{|c|c|c|c|c|c|c|c|}
\hline Futteraufnahme (kg TM $\left.{ }^{\top} / \mathrm{Tag}\right)$ & $\mathrm{N}$ & Mittel & $\sigma_{p}^{(2)}$ & VK $(\%)$ & $h^{2}$ & & $\sigma_{\mathrm{A}}$ \\
\hline 112. - 162. Lebenstag & 182 & 3,4 & 0,47 & 13,8 & 0,82 & 0,31 & 0,43 \\
\hline 163, - 212. Lebenstag & 262 & 4,6 & 0,58 & 12,6 & 0,24 & 0,21 & 0,28 \\
\hline 213. - 262. Lebenstag & 269 & 5,7 & 0,65 & 11,4 & 0,09 & 0.16 & 0,20 \\
\hline 263. - 312. Lebenstag & 269 & 6,5 & 0,75 & 11,5 & 0,65 & 0,23 & 0,60 \\
\hline 112. - 312. Lebenstag & 269 & 5,1 & 0,54 & 10,6 & 0,42 & 0,20 & 0,35 \\
\hline
\end{tabular}

Sowohl die genetische als auch die phänotypische Korrelation zwischen der Futteraufnahme im 4. Teilabschnitt und dem Gesamtabschnitt waren mit 0,88 bzw. 0,85 hoch (Tab. 5).

\section{Tabelle 5}

Genetische (oberhalb der Diagonalen) und phănotypische (unterhalb der Diagonalen) Korrelationen (Standardfehler als Subskript) zwischen der Futteraufnahme in aufeinanderfolgenden Prufabschnitten potentieller Besamungsbullen (Genetic (above diagonal) and phenotypic (below diagonal) correlation (standard error in subscript) between feed intake in successive test periods in potential Al bulls)

\begin{tabular}{|c|c|c|c|c|c|c|c|c|c|}
\hline & Futteraufnahme (kg TM'/Tag) & $1^{2}$ & $2^{2}$ & $3^{2}$ & & $4^{2}$ & & $5^{2}$ & \\
\hline 1 & 112. - 162. Lebenstag & & $\mathrm{NK}^{3}$ & 0,99 & 0,52 & 0,24 & • & 0,77 & 0,13 \\
\hline 2 & 163. -212 . Lebenstag & 0,60 & & $\mathrm{NK}^{3}$ & & 0,65 & 0,22 & 0,96 & 0,08 \\
\hline 3 & 213. - 262. Lebenstag & 0,49 & 0,68 & & & 0,99 & 0,11 & $\mathrm{NK}^{3}$ & \\
\hline 4 & 263. - 312. Lebenstag & 0,41 & 0,48 & 0,77 & & & & 0,88 & 0.07. \\
\hline$\ddot{5}$ & $112 .-312$. Lebenstag & 0,68 & 0,81 & 0,93 & & $0,85^{\circ}$ & & & \\
\hline
\end{tabular}

Trockenmasse, ${ }^{2}$ Die Ziffern stehen fur die Merkmale in der 1. Spalte. 'Nicht konvergiert

4.

Diskussion

Für die mittlere Merkmalsausprägung im Gesamtabschnitt kann eine gute Übereinstimmung mit Ergebnissen aus der Literatur beobachtet werden. Die Untersuchung von STAMER et al. (1996) basierte auf teilweise den gleichen Daten wie in der vorliegenden Analyse und ergab eine tägliche Futteraufnahme von $5 \mathrm{~kg}$ und eine Futteraufnahmedauer von 140 Minuten pro Tag. STAMER et al. (1997) berechneten eine Futteraufnahmedauer von 119 Minuten und HARTMANN (1989) fand einen Wert von 140 Minuten pro Tag. 
Die in dieser Analyse geschätzte Heritabilität von 0,42 liegt im oberen Bereich der Schätzwerte in der Literatur (WASSMUTH, 1999). Für die Futteraufnahmedauer sind nur wenige Schätzwerte in der Literatur zu finden. MOKHOV (1983) schätzte eine Heritabilität von 0,68, die deutlich über der in dieser Arbeit gefundenen Erblichkeit der Futteraufnahmedauer von 0,40 liegt. Aufgrund der Ergebnisse kann von einer mittleren Heritabilität der Futteraufnahmedauer ausgegangen werden.

In der Tendenz bestätigt die Literatur eine enge genetische Beziehung zwischen der Futteraufnahme und der täglichen Zunahme, die in der vorliegenden Analyse gefunden wurde. JENSEN et al. (1995) schätzten genetische Korrelationen zwischen der Futteraufnahme und der Wachstumsgeschwindigkeit zwischen 0,63 und 0,75 in Abhängigkeit von der Fütterungsintensität. Auch FAN et al. (1995) veröffentlichten ähnlich hohe Korrelationen zwischen 0,76 und 0,89 für verschiedene Rassen. Die von HARTMANN (1989) geschätzten phänotypischen Korrelationen waren mit 0,47 bis 0,52 niedriger als die genetischen Korrelationen und auch niedriger als die hier gefundene phänotypische Beziehung.

Allerdings darf die hohe genetische Korrelation zwischen der Futteraufnahme und der täglichen Zunahme nicht zur Substitution der Futteraufnahmeprüfung führen. Wie WASSMUTH (1999) zeigen konnte, besteht eine mittlere genetische Korrelation zwischen der Futteraufnahme von Besamungsbullen und der Disposition ihrer laktierenden Töchter an Ketose zu erkranken. Auch LYNCH et al. (1992) sehen einen engen Zusammenhang zwischen der Gesundheit und der Futteraufnahme, da das Immunsystem über den Hypothalamus die Futteraufnahme indirekt beeinflußt. Mithin kann die Futteraufnahme als Indikator der Gesundheit von Milchkühen züchterisch genutzt werden.

Die tägliche Zunahme sollte nicht als Indikator der Gesundheit herangezogen werden, da auch subklinisch erkrankte Tiere hohe Wachstumsleistungen erbringen können.

Die Ergebnisse der vorliegenden Untersuchung deuten an, daß keine enge Beziehung zwischen der Futteraufnahme und der Futteraufnahmedauer besteht. Diese Beobachtung wird durch die Mehrzahl der Literaturergebnisse bestätigt. HARTMANN (1989) fand eine niedrige phänotypische Korrelation von 0,30 und auch STAMER et al. (1996) schätzten mit 0,31 eine niedrige Korrelation. An Kühen schätzte STAMER (1995) eine Korrelation von 0,39. Eine auffallend hohe phänotypische Korrelation von 0,72 berechneten JACKSON et al. (1991).

Auch NIELSEN (1999) zieht aus ihrer Literaturübersicht den Schluß, daß das Futteraufnahmeverhalten nicht zwangsläufig mit der Futteraufnahme in enger Beziehung stehen muß, sondern von dem Sozialgefüge innerhalb der Gruppe und dem Tier:Freßplatz-Verhältnis abhängt. Deshalb kann das Verhalten bei der Futteraufnahme ein Indikator der sozialen Belastung, der ein Tier innerhalb einer Gruppe ausgesetzt ist, sein und Hinweise auf die Tiergerechtheit eines Haltungssystems geben.

Die Untersuchungen von KROHN und KONGGAARD (1976) bestätigen diese Hypothese durch die Beobachtung, daß der soziale Rang einer Kuh innerhalb der Gruppe das Futteraufnahmeverhalten beeinflußte. Kühe mit einem höheren Rang nahmen eine größere Futtermenge auf und hatten eine kürzere Futteraufnahmedauer am Freßgitter. HARB et al. (1985) beobachteten eine deutlichere Steigerung der Futteraufnahmegeschwindigkeit bei Kühen mit einem niedrigen Rang im Vergleich zu Kühen 
mit einem hohen Rang bei Verringerung der Freßplätze von 10 auf 5 in einer Gruppe mit 10 Kühen. Auch in der Untersuchung von COULON et al. (1987) hatte der soziale Rang einen signifikanten Einfluß auf die aufgenommene Futtermenge.

Aus organisatorischen Gründen konnten nur $68 \%$ aller angelieferten Jungbullen vom 112. Lebenstag an gepruft werden. Bei den restlichen Tieren erstreckte sich die Anlieferung bis zum 160 . Lebenstag. Deshalb kann furr eine Teilabschnittsprüfung weder der 1. noch der 2. Abschnitt empfohlen werden.

Es konnte beobachtet werden, daß sowohl die mittlere Futteraufnahme als auch deren Variation vom 1. bis zum 4. Teilabschnitt stiegen und der Variationskoeffizient nur tendenziell geringer wurde. Im 4. Teilabschnitt betrug der Variationskoeffizient noch $11,5 \%$. In der Analyse von RUNNWERTH et al. (1991) variierte die Futteraufnahme zwischen $3,7 \mathrm{~kg}$ TM/Tag und 7,8 kg TM/Tag und der Variationskoeffizient schwankte zwischen 4,0 \% und 10,9\%, was die eigenen Ergebnisse weitestgehend untermauert. Einen deutlich höheren Wert von 16,7 \% berechnete HARTMANN (1989) für die Futteraufnahme von aufgeschlossenen Strohcobs. Der Unterschied zu dem in der vorliegenden Untersuchung gefundenen Wert, dürfte in dem energieärmeren Grundfutter zu sehen sein. Letzteres dient der Erfassung der kapazitiven Futteraufnahme, die einen Indikator für das Volumen der Vormägen darstellt. Die Futteraufnahme, die mit Prufdiäten einer höheren Verdaulichkeit der organischen Substanz erfaßt werden, unterliegen stärker der physiologischen und weniger der physikalischen Regulation (CONRAD et al., 1964).

Im vierten Teilabschnitt sind sowohl die Heritabilităt als auch die genetische Standardabweichung höher als die geschätzten Werte für den Gesamtabschnitt. Mithin erscheint der Teilabschnitt 4 als empfehlenswert für eine Verkürzung der Futteraufnahmeprüfung. RUNNWERTH et al. (1991) kommen ebenfalls zu dem Schluß, daß die Futteraufnahmeprüfung verkürzt werden kann. Die Autoren folgern dies aus der Beobachtung mittlerer bis hoher Korrelationen zwischen Einzelprüfzeiträumen und der gesamten Prüfzeit.

Auch die hohen genetischen und phänotypischen Korrelationen lassen den Schluß zu, daß eine Futteraufnahmeprüfung vom 263. bis 312. Lebenstag ein guter Indikator für die Futteraufnahme vom 112. bis 312 . Lebenstag ist.

Diese Aussage wird durch die Ergebnisse von HARTMANN (1989) bestätigt. Der Autor fand bei der Untersuchung von Beziehungen zwischen verschiedenen Altersabschnitten und dem Gesamtabschnitt die höchste phänotypische Korrelation zwischen dem Altersabschnitt 263. bis 312. Lebenstag und dem Gesamtabschnitt 112. bis 540 . Lebenstag. WASSMUTH (1996) konnte mittels der hohen phänotypischen Korrelation von 0,93 nachweisen, daß die Futteraufnahme in der Langzeitprüfung vom 112. bis 540. Lebenstag eine enge Beziehung zur Kurzzeitprufung vom 130. bis 330. Lebenstag besitzt.

Für das Prüfmerkmal tägliche Zunahme muß von einer Verkürzung der Prüfperiode abgeraten werden, da die genetische Korrelation zwischen der Wachstumsleistung im 4. Teilabschnitt (263. bis 312. Lebenstag) und dem Gesamtabschnitt (122. bis 312. Lebenstag) nur 0,70 betrug. HARTMANN (1989) sowie LANGHOLZ und JONGELING (1972) fanden deutlich geringere Korrelationen. Letztere folgerten aus ihren Untersuchungen, daß unterschiedliche Genwirkungen das Wachstum in verschiedenen 
Altersabschnitten steuern.

Demnach hat die Prüfung der täglichen Zunahme über mindestens 200 Tage zu erfolgen und sollte nicht verkürzt werden. Für die Futteraufnahmeprüfung hat ein 200-tägiger Aufenthalt in der Station den Vorteil, daß während der ersten 150 Tage die Jungbullen eine Anpassung an die Stationsbedingungen und besonders an die Prüfdiät durchlaufen. Somit gelingt es, den Einfluß der Herkunftsherde auf die Futteraufnahme weitestgehend auszuschließen.

\section{Schlußbetrachtung}

Die stationäre Futteraufnahmeprüfung potentieller Besamungsbullen kann zur züchterischen Verbesserung der Gesundheit ihrer milchleistenden Kühe beitragen. Aus diesem Grunde kann sie auch in Zukunft eine wichtige Form der Leistungsprüfung bei Rindern sein. Darüber hinaus ist die Erfassung der Futteraufnahme an potentiellen Bullenmüttern in kontrollierten Nukleusherden möglich und sollte zukünftig in Betracht gezogen werden.

In den 4 Bullenprüfstationen mit Erfassung der Futteraufnahme erstreckt sich die Prüfung auf einen Zeitraum von 200 bis 265 Tagen. Die vorliegende Untersuchung wurde durchgeführt, um Möglichkeiten der Kostensenkung zu prüfen.

Aus den Ergebnissen kann gefolgert werden, daß eine zeitliche Verkürzung der Futteraufnahmeprufung ohne einen größeren Informationsverlust erfolgen kann. Vorzugsweise sollten die letzten 50 Tage der bisherigen Prufperiode zur Erfassung der Futteraufnahme genutzt werden. Zur weitgehenden Reduktion des Effektes der Herkunftsherde auf die Futteraufnahme muß eine entsprechend lange Vorbereitungsphase, während der sich die Jungbullen an die Stationsumwelt gewöhnen können, vorgeschaltet werden.

Aufgrund der vorliegenden Ergebnisse muß von einer indirekten Erfassung der Futteraufnahme mittels des Futteraufnahmeverhaltens abgeraten werden. Allerdings ist bei dieser Aussage das für genetische Parameterschätzungen geringe Datenmaterial zu berilcksichtigen. Es gilt, die hier gefundenen Resultate an einer größeren Tierzahl zu überprüfen.

\section{Literatur}

ANDERSEN, O.:

Health control.In: Summary of the annual report 1991, National Committee on Danish Cattle Husbandry, 9 Seiten. 1991

ANONYMUS:

Sire evaluation procedures for non-dairy-production and growth \& beef production traits practised in various countries 1996. International Bull Evaluation Service, Bulletin No. 13. 1996

BRANDT, A.; PAPST, K.; SCHULTE-COERNE, H.; GRAVERT, H.O.:

Die Heritabilităt der Futteraufnahme bei Milchkulhen. Zulchtungskunde, Stuttgart 57 (1985), 299-308

CONRAD, H.R.; PRATT, A.D.; HIBBS, J.W.: Regulation of feed intake in dairy cows. I. Change in importance of physical and physiological factors with increasing of digestibility. J. Dairy Sci., 47 (1964), 54-62

COULON, J.B.; DOREAU, M.; REMOND, B.; JOURNET, M.:

Evolution des activités alimentaires des vaches laitières en début de lactation et liaison avec les quantités d'aliments ingérées. Reprod. Nutr. Dévelop., 27 (1987), 67-75 
FAN, L.Q.; BAILEY, D.R.C.; SHANNON, N.H.:

Genetic parameter estimation of postweaning gain, feed intake, and feed efficiency for Hereford and Angus bulls fed two different diets. J. Anim. Sci., 73 (1995), 365-372

GROEN, A.F.; HELLINGA, I.; OLDENBROEK, J.K.:

Genetic correlations of clinical mastitis and feet and legs problems with milk yield and type traits in Dutch Black and White dairy cattle. Netherlands Journal of Agricultural Science, 42 (1994) 4, 371-378

GROEN, A.F.; STEINE, T.; COLLEAU, J.-J.; PEDERSEN, J.; PRIBYL, J.; REINSCH, N.: Economic values in dairy cattle breeding, with special reference to functional traits. Report of an EAAP-working group. Livestock Production Science 49 (1997), 1-21

GROENEVELD, E.; GARCIA CORTÉS. A.:

VCE 4.0, a (co)variance components package for frequentists and bayesians. Proceedings of the $6^{\text {th }}$ World Congress on Genetics Applied to Livestock Production, Armidale, 1998, Vol. 27, 455-456

HARB, M.Y.; REYNOLDS, V.S.; CAMPLING, R.C.:

Eating behaviour, social dominance and voluntary intake of silage in group-fed milking cattle. Grass Forage Sci. 40 (1985), 113-118

HARTMANN, J.:

Untersuchungen zur Automatisierung der Futterung in der stationåren Nachkommenprüfung auf Fleischleistung beim Rind. Universität Göttingen, Diss., 1989

JACKSON, D.A.; JOHNSON, C.L.; FORBES, J.M.:

The effect of compound composition and silage characteristics on silage intake, feeding behaviour, production of milk and live-weight change in lactating dairy cows. Animal Production 52 (1991), 11-19

JAKOBSEN, J.H.:

Genetic and phenotypic parameters for traits measured on performance test bulls and the genetic correlation to their daughters yield. M.Sc. Speciale I Speciel Husdyravl, L 8332, Insitut for Husdyrbrug og Husdyrsundhed, Den kgl. Veterinær og Landbohøjskole, København, April 1995

JENSEN, J.; HOHENBOKEN, W.D.; MADSEN, P.; ANDERSEN, B.B.:

Sirexnutrition interactions and genetic parameters for energy intake, production and efficiency of nutrient utilization in young bulls, heifers and lactating cows. Acta Agric. Scand., Sect. A, Animal Sci., 45 (1995), 81-91

KROHN, C.C.; KONGGAARD, S.P.:

Investigations concerning feed intake and social behaviour among group fed cows under loose housing conditions. 441. Beretning fra Statens Husdyrbrugsforsøg. (1976)

LANGHOLZ, H.-J.; JONGELING, C.:

Untersuchungen zum genetischen Aussagewert der stationăren Nachkommenprüfung auf Mastleistung und Schlachtkörperwert beim Rind. Züchtungskunde, Stuttgart 44 (1972), 368-384

LYNCH, J.J.; HINCH, G.N.; ADAMS, D.B.:

The behaviour of sheep. Biological principles and implications for production. CAB International, Wallingford, UK, 1992

MADSEN, P.; PEDERSEN, G.A.; JENSEN, J.:

Multibreed evaluation of bulls performance tested for growth and feed intake based on full and part test period records. Proc. of the $46^{\text {th }}$ Meeting of the European Association for Animal Production, Prague, Czech Republic 4 -7 September, 1995

MÄNTYSAARI, E.A.; GRÖHN, Y.T.; QUAAS, R.L.:

Clinical ketosis: phenotypic and genetic correlations between occurrences and with milk yield. J. Dairy Sci., 74 (1991), 3985-3993

MOKHOV, B.P.:

(Breeding of cattle for stereotyped behaviour). Doklady Vsesoyuznoi Akademi Sel ${ }^{*}$ skokkozyoistvennykh Nauk, 9, 32-35, (1983). Zitiert nach: PHILLIPS, C.J.C.: Cattle behaviour, $1^{\text {st }}$ Edition, Farming Press Books, Ipswich, UK, 212 pp., 1993

NEUMAIER, A.; GROENEVELD, E.: Restricted maximum likelihood estimation of covariances in sparse linear models. Genet. Sel. Evol., 30 (1998), 3-26

NIELSEN, B.L.:

On the interpretation of feeding behaviour measures and the use of feeding rate as an indicator of social constraint. Applied Animal Behaviour Science 63 (1999), 79-91

NIEUWHOF, G.J.; VAN ARENDONK, J.A.M., VOS, H.; KORVER, S.: Genetic relationships between feed intake, efficiency and production traits in growing bulls, growing heifers and lactating heifers. Livest. Prod. Sci., 32 (1992), 189-202 


\section{POTTHAST, J.:}

Untersuchungen zur Integration funktionaler Selektionsmerkmale in große Besamungszuchtprogramme POTUCEK, E.: bei Milchrindern. Universităt Bonn, Diss., 1999

Schätzung genetischer Parameter und Genotyp-Umwelt-Interaktionen für Mast- und Schlachtleistungskriterien beim Fleckvieh auf Station. Universitut Wien, Diss., 1990

PRYCE, J.E.; VEERKAMP, R.F.; THOMPSON, R.; HILL, W.G.; SIMM, G.: Genetic aspects of common health disorders and measures of fertility in Holstein Friesian dairy cattle. Anim. Sci., 65 (1997), 353-360

RUNNWERTH, E.; ZELFEL, S.; KREMPA, T; JAZDZEWSKI, J.: Zuchterische Moglichkeiten einer Selektion auf Futteraufnahme und Futteraufwand bei Zweinutzungsund Fleischrindbullen. 1. Mitt.: Prufung auf Futteraufnahme. Arch. Tierz., Berlin 34 (1991) 2, 115-124

\section{SAS INSTITUTE INC.} SAS/STAT User's Guide, Version 6, $4^{\text {th }}$ Edition, Volume 2.

SIMIANER, H.; SOLBU, H.; SCHAEFFER, L.R.: Estimated genetic correlations between disease and yield traits in dairy cattle. J. Dairy. Sci., 74 (1991),
$4358-4365$

STAMER, E.: Futteraufnahmeverhalten von Milchkuhen - Analyse der Zeitstruktur und Nutzungsmoglichkeiten.
Universităt Kiel, Diss., 1995

STAMER, E.; REINSCH, N.; JUNGE, W.; ALPS, H.; KALM, E.:

Beziehungen zwischen Futteraufnahmeverhalten und Verzehr bei Jungbullen. Vortrag anläßlich der Sitzung des Arbeitskreises „Leiter der Fleischleistungsprufanstalten beim Rind" am 21./22. Oktober 1996 in Trappenkamp

STAMER, E.; REINSCH, N.; JUNGE, W.; HASENPUSCH, E.; KALM, E.:

Stand der Freßwertschätzung in der ELP-Station Ruhwinkel. Vortrag anlaßlich der Sitzung des Arbeitskreises ,Leiter der Fleischleistungsprufanstalten beim Rind" am 21./22. Oktober 1997 in Meißen

URIBE, H.A.; KENNEDY, B.W.; MARTIN, S.W.; KELTON, D.F. Genetic parameters for common health disorders of Holstein cows. J. Dairy Sci., 78 (1995), $421-430$

VAN ARENDONK, J.A.M., NIEUWHOF, G.J., VOS, H.; KORVER, S.:

Genetic aspects of feed intake and efficiency in lactating dairy heifers. Livest. Prod. Sci., 29 (1991), 263-275

WASSMUTH, R.:

Feed intake capacity as selection criterion in dairy cattle. Proc. 47. Jahrestagung der EVT in Lillehammer/Norwegen vom 25. bis 29. August 1996, C 3.8.

WASSMUTH, R::

Die stationäre Futteraufnahmeprufung von Bullen als Indikator der Gesundheit bei Milchkühen. Habilitationsschrift, Fakultăt für Agrarwissenschaften, Universität Göttingen, Cuvillier Verlag Gottingen, ISBN 3-89712-685-0, 1999

WASSMUTH, R.; ALPS, H.:

Stand der stationären Fleischleistungsprufung beim Rind in Deutschland. Züchtungskunde, Stuttgart 67 (1995), 185-205

Eingegangen: 20.04.2000

Akzeptiert: 27.09 .2000

Anschriften der Verfasser

PD Dr. RALF WASSMUTH

Institut für Tierzucht und Haustiergenetik

Georg-August-Universităt Göttingen

Albrecht-Thaer-Weg 3

D-37075 Gottingen

E-Mail: rwassmu@gwdg.de

Dr. HEINO ALPS

Hessische Landesanstalt fur Tierzucht

Neu-Ulrichstein

D-35315 Homberg/Ohm 


\section{POSITION ANNOUNCEMENT}

At the Research Institute for the Biology of Farm Animals (FBN) Dummerstorf, Germany

Department of Genetics and Biometry (web-site: http://www.fbn-dummerstorf.de) following position is available:

\section{SCIENTIFIC ASSISTANT or PhD-STUDENT in MOLECULAR GENETICS}

to support a DFG (German Research Council) funded project:

"Evaluation of extreme growth lines of laboratory mice for construction and using resource families to identify genetic factors of growth components"

The position is funded for 1.3 years with a salary according to BAT IIa/2.

Starting-date: as soon as possible.

Requirements:

- university degree in molecular genetics or a closely related field

Following skills are expected:

- strong background in current methodology in DNA-technology

- Knowledge in general animal breeding and QTL strategies

- Experiences in handling modern software and in biostatistical methods

Working place will be the Research Institute for Biology of Farm Animals in Dummerstorf (near Rostock $(10 \mathrm{~km}$ ) in Mecklenburg - Vorpommern close to the coast of the Baltic Sea)

Interested candidates should send their detailed application including a CV to:

Research Institute for Biology of Farm Animals

Department of Genetics and Biometry

Dr. U. Renne

Wilhelm-Stahl-Allee 2

D-18196 Dummerstorf, Germany

Further information could be obtained by e-mail: renne@fbn-dummerstorf.de 